\title{
EVOLUTION OF DIRAC CONE IN DISCLINATED GRAPHENE
}

\author{
M.A. Rozhkov ${ }^{1}$, A.L. Kolesnikova, ${ }^{1,2}$ I. Hussainova ${ }^{1,3}$, M.A. Kaliteevskii', \\ T.S. Orlova ${ }^{1,4}$, Yu.Yu. Smirnov ${ }^{1}$, I.S. Yasnikov ${ }^{5}$, L.V. Zhigilei ${ }^{1,6}$, V.E. Bougrov ${ }^{1}$ \\ and A.E. Romanov ${ }^{1,4}$
}

\author{
${ }^{1}$ ITMO University, St. Petersburg, Russian Federation \\ Institute of Problems of Mechanical Engineering, RAS, St. Petersburg, Russian Federation \\ ${ }^{3}$ Tallinn University of Technology, Tallinn, Estonia \\ ${ }^{4}$ loffe Institute, RAS, St. Petersburg, Russian Federation \\ ${ }^{5}$ Togliatti State University, Togliatti, Russian Federation \\ ${ }^{6}$ Department of Materials Science \& Engineering, University of Virginia, Charlottesville, USA
}

Received: May 05, 2018

\begin{abstract}
Graphene crystals, containing arrays of disclination defects, are modeled and their energies are calculated using molecular dynamics (MD) simulation technique. Two cases are analyzed in details: (i) pseudo-graphenes, which contain the alternating sign disclination ensembles and (ii) graphene with periodic distribution of disclination quadrupoles. Electronic band structures of disclinated graphene crystals are calculated in the framework of density functional theory (DFT) approach. The evolution of the Dirac cone and magnitude of band gap in the band structure reveal a dependence on the density of disclination quadrupoles and alternating sign disclinations. The electronic properties of graphene with disclination ensembles are discussed.
\end{abstract}

\section{INTRODUCTION}

Carbon materials such as graphene, fullerenes and nanotubes, as well as composites based on them, are promising nanomaterials: they demonstrate high electrical conductivity [1], remarkable thermal conductivity [2], and unique strength [3].

Graphene is one of the so-called Dirac cone materials, which has a cone-like band structure with linear dispersion near K (or K') point [4] (Fig. 1). The Hamiltonian near K (or K') is identical to that of massless Dirac equation, therefore $\mathrm{K}$ or $\mathrm{K}$ ' points are called Dirac points, and the linear band structure near them is named as Dirac cone [4]. Dirac cone structure dictates the existence of massless fermions in graphene, leading to many specific properties such as quantum Hall effects and ultrahigh carrier mobility, see, for example, [4-6]. At present, graphene is the only $2 \mathrm{D}$ material with experimentally confirmed Dirac cone $[5,6]$.

Through external influence and by introducing defects into the crystal lattice, it is possible to control graphene properties. For example, computer simulations have shown that tensile deformation opens the bang gap at graphene [7], and the defects of the crystal lattice can change its mechanical properties $[8,9]$ contributing in the same time to the increase in electrical $[10]$ and thermal $[8,11]$ conductivities.

In a graphene crystal, impurity atoms [12], carbon rings with locally broken axial hexagonal symmetry [12-14], as well as ensembles of such rings forming grain boundaries and intercrystallite boundaries $[10,15]$, can be viewed as disclination defects. Carbon rings in the form of squares, pentagons,

Corresponding author:A.E. Romanov, e-mail: alexey.romanov@niuitmo.ru 

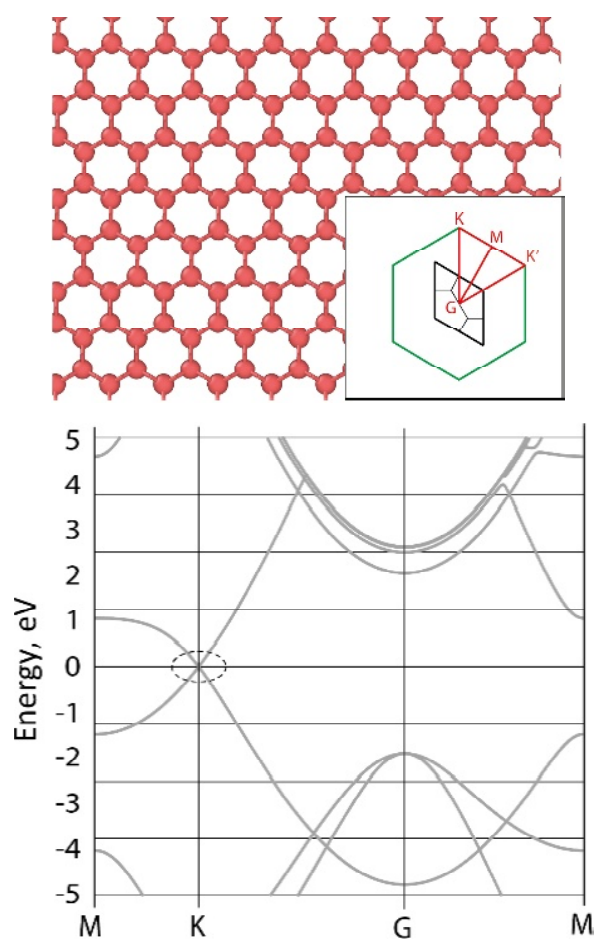

Fig. 1. Graphene crystal and its band structure. In the inset for graphene crystal schematics, Brillouin zone is shown. Dirac cone is outlined by a dashed ellipse.

heptagons, and octagons are the cores of wedge disclinations with a power of $+2 \pi / 3,+\pi / 3,-\pi / 3$, and $-2 \pi / 3$, respectively [16-18]. Fig. 2 shows a geometric scheme of the formation of wedge disclinations of power $+\pi / 3$ and $-\pi / 3$ in a graphene crystal $[17,18]$.
A single disclination introduces global elastic distortions into the crystal [19]. As a result, in real crystals, disclinations can occur as ensembles quadrupoles, grain boundaries and intercrystallite boundaries [17,18,20,21], and as networks composed of disclinations of opposite signs $[17,18,22,23]$. Densely packed with disclinations, graphene crystals contain a small number of hexagons typical of graphene, or do not contain them at all. Such carbon based crystalline objects acquired special names: phagraphene [22] consisting of pentagons, heptagons and hexagons; PO-graphene [23], consisting of pentagons and octagons; and others generalized by the concept of pseudographenes [17,18].

It has been shown in Ref. [22] that phagraphene possesses specific potential energy (i.e. energy per atom) $E_{\mathrm{ph}}$, slightly exceeding the energy of graphene $E_{\mathrm{Ph}}-E_{\mathrm{Graph}} \approx 0.2 \mathrm{eV} /$ atom and, similar to graphene, its band structure has Dirac cone, compare Fig. 1 and Fig. 3a. PO-graphene band structure also demonstrates the presence of Dirac cone, but in this case the Dirac cone is displaced with respect to the Fermi level [23] (Fig. 3b).

Despite the available papers [22,23], no systematic analysis of the influence of disclination defects on the graphene band structure has been carried out. In this paper, we present a study of the effect of the density of uniformly distributed disclinations with opposite signs (disclination networks - DNs) and the density of disclination quadrupole networks DQNs on the average energy per atom and the band

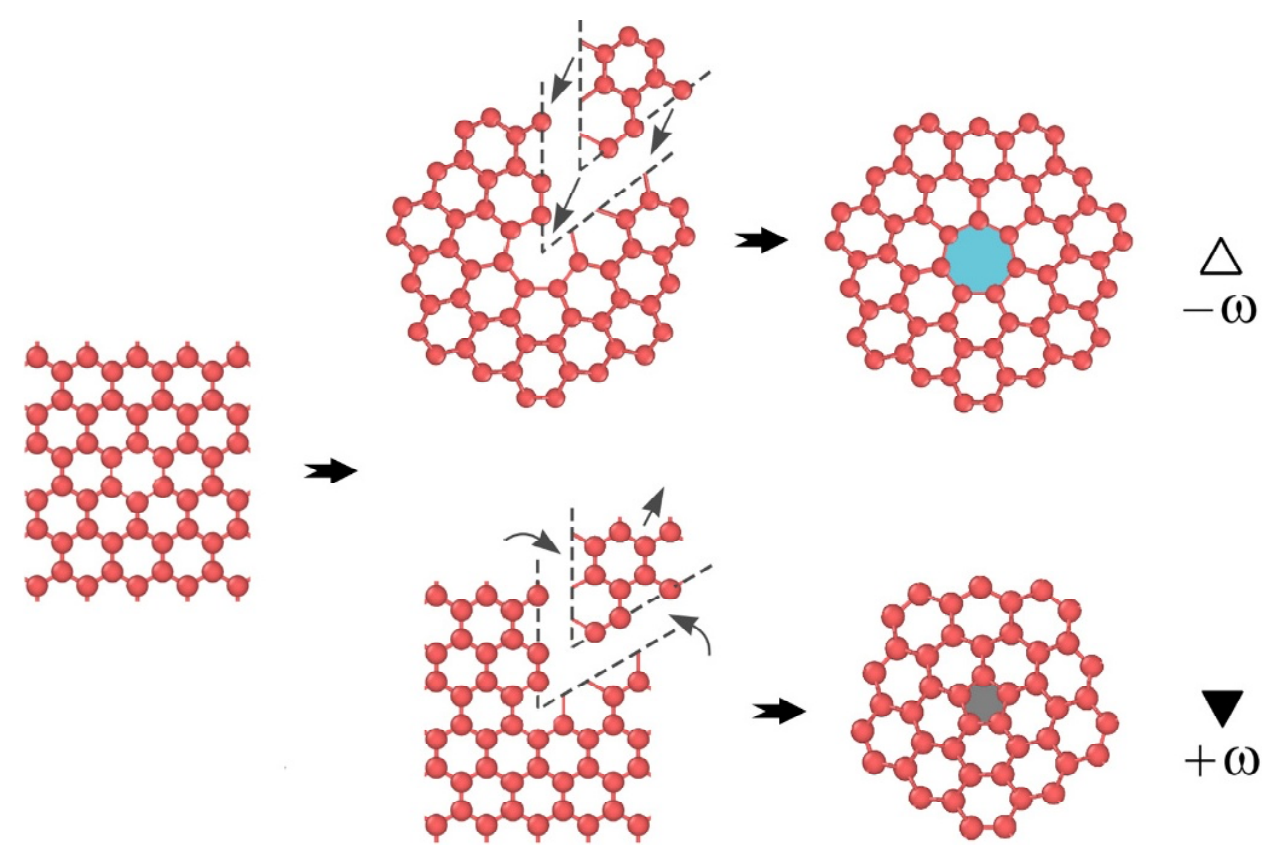

Fig. 2. Geometrical scheme of the disclination formation in graphene. The magnitude of disclination power is $\omega=\pi / 3$. 

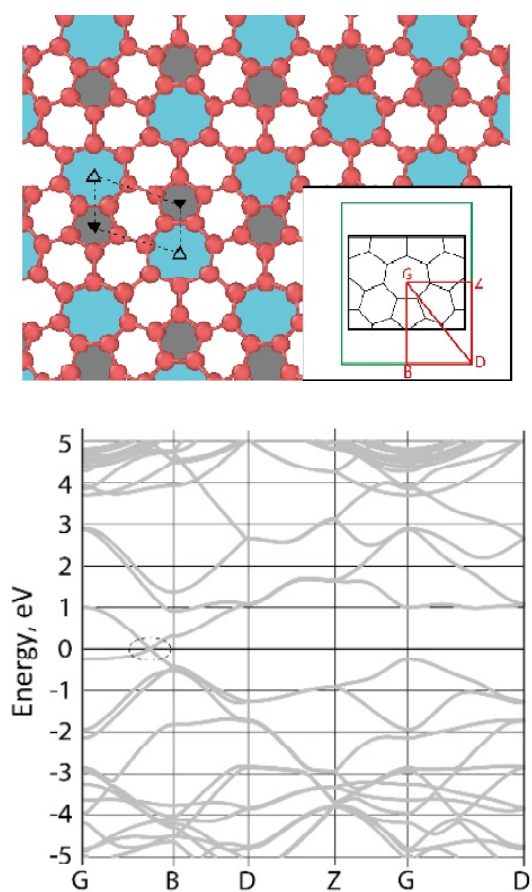

(a) Phagraphene (5-7A)
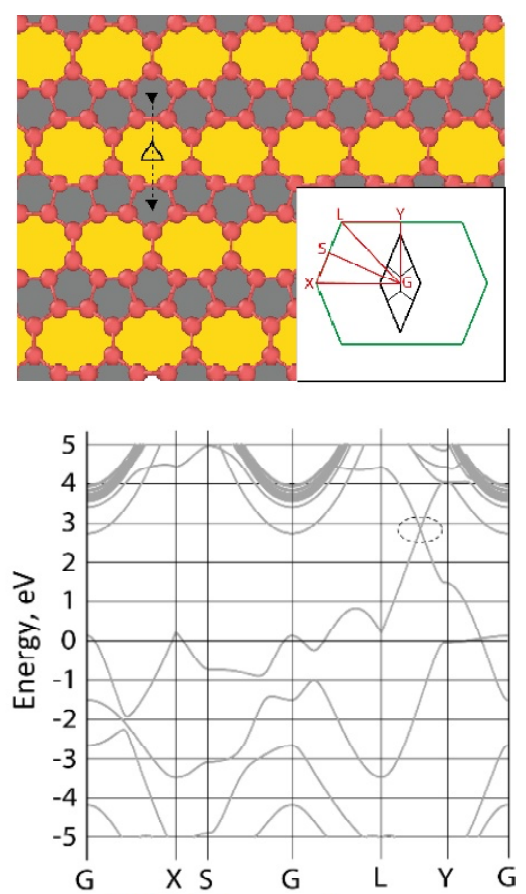

(b) PO-graphene

Fig. 3. Phagraphene (a) and PO-graphene (b) and their band structures (replotted using the data reported in $[22,23])$. In crystal structures, the disclination quadrupoles are depicted, and the insets show Brillouin zones. Dirac cones are outlined by dashed ellipses.

structure of disclinated graphene utilizing the molecular dynamics (MD) technique and density functional theory (DFT) approach, respectively.

\section{MODELING TECHNIQUES}

To design and optimize the geometry of graphene and graphene crystals with disclinations, we applied MD modeling technique implemented in the LAMMPS software package [24]. For our problem, the AIREBO interatomic interaction potential was used [25]. The algorithm of the conjugate PolakRibiera gradient at OK [26] was carried out for the energy minimization of graphene.

To calculate the band structures of graphene, the DFT based CASTEP software package [27] with generalized gradient approximation (GGA) was exploited using the Purdue-Burke-Ernzerhof exchangecorrelation functional (PBE) [28].

\section{ENERGETICS AND BAND STRUCTURES OF DISCLINATED GRAPHENE. RESULTS AND DISCUSSION}

In Fig. 4, the modeled graphene crystals with disclinations of powers $\pm \pi / 3$, i.e. with pentagonal and heptagonal carbon rings, periodically distributed over graphene, are presented. Here, crystals with carbon pentagons and heptagons we mark as 5-7. Phagraphene is denoted as 5-7A (Fig. 3a); crystal with the most densely packed disclinations is denoted as 5-7B (Fig. 4a); two other crystals are 57Bg1 and 5-7Bg2 (Fig. 4b,c).

Crystals 5-7A, 5-7B, 5-7Bg 1 and 5-7Bg2 can be considered, on the one hand, as embedded in graphene DNs and, on the other hand, as pseudographenes $[17,18]$ containing or not containing hexagons. In Fig. 4, disclination quadrupoles of DNs are indicated in atomic structures of crystals.

The average specific potential energies of crystals 5-7B, 5-7Bg1, and 5-7Bg2 do not exceed significantly the energies of graphene and phagraphene (Fig. 5). Therefore, synthesis of such pseudographenes may be possible in the future.

It follows from the results given in Fig. 4 that with an increase in the period of the DNs, i.e. with an increase in the number of elastically distorted hexagons in pseudo-graphenes, their band structures undergo significant changes. Namely, the Dirac cone disappears, and the valence and conduction bands overlap, albeit slightly. Band gaps for the crystals 5-7B, 5-7Bg1, and 5-7Bg2 are zero.

Adding the hexagons to the pseudo-graphene 5-7B can be done differently than described above. If we surround disclination quadrupoles with hexagons, without changing the characteristic dimen- 


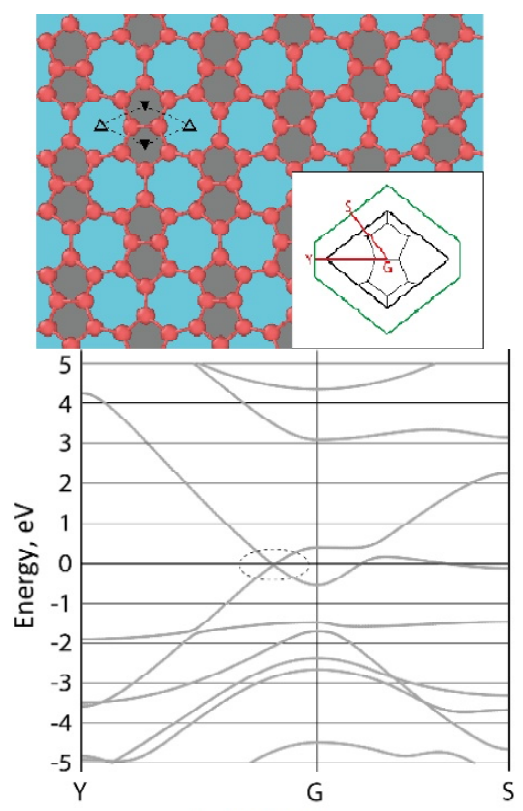

(a) $5-7 \mathrm{~B}$

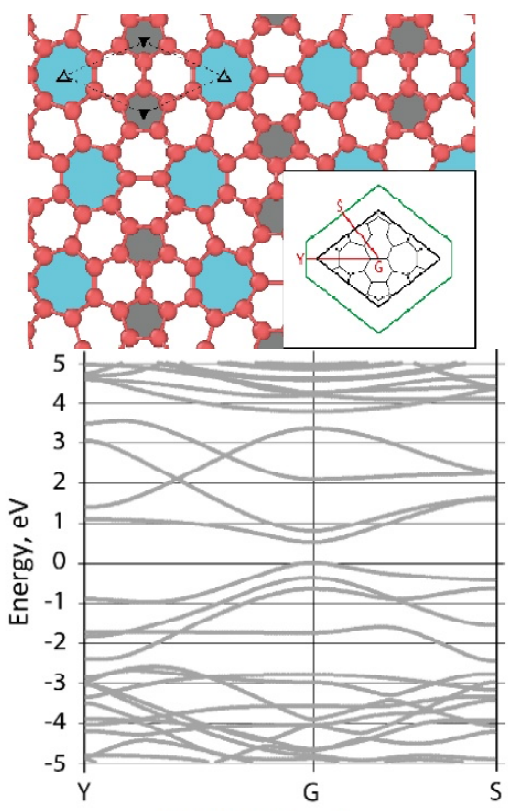

(b) $5-7 \mathrm{Bg} 1$

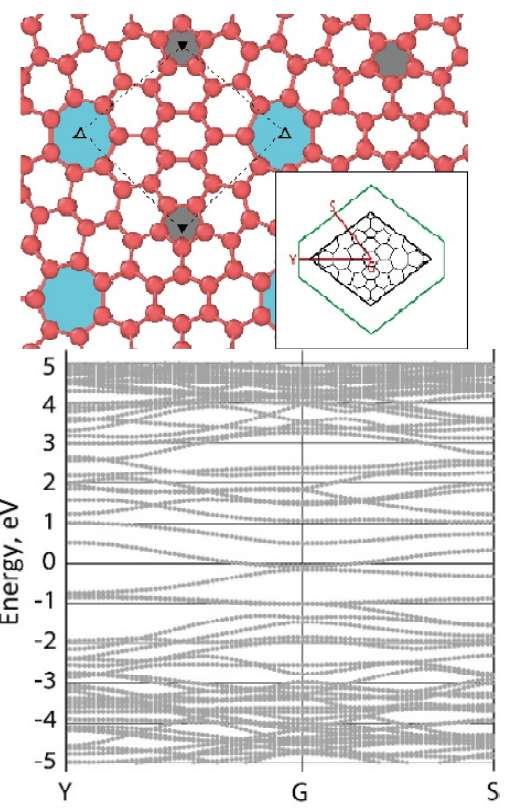

(c) $5-7 \mathrm{Bg} 2$

Fig. 4. Pseudo-graphenes designed by disclination networks and their band structures. In crystal lattices, the disclination quadrupoles are depicted, and the insets show Brillouin zones. Pseudo-graphenes are marked with 5-7B, 5-7Bg1, and 5-7Bg2. In band structure of 5-7B (a), Dirac cone in (a) is outlined by dashed ellipse.

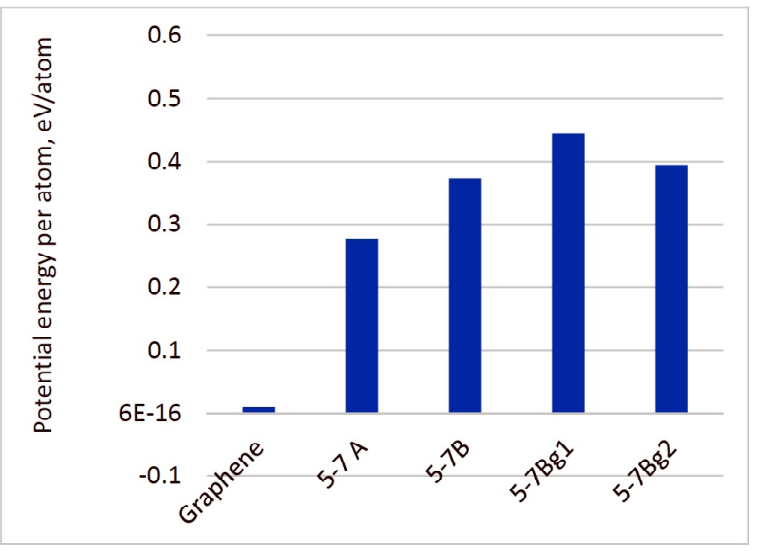

Fig. 5. Average specific potential energies (energies per atom) of studied pseudo-graphenes.

sions of the quadrupoles, we obtain the networks of disclination quadrupoles - DQNs shown in Fig. 6.

It is known that the longer the period of such DQN, the more undistorted hexagons appear in the crystal. This is due to the fact that the disclination quadrupole is a self-screening defect configuration; the elastic distortions induced by a quadrupole in the crystal lattice decrease rapidly and become insignificant at distances of about 3-5 quadrupole sizes [19]. It is clear that increasing the period of the DQN, it is possible to come to the limit of ideal graphene in the large areas of the crystal. Therefore, the band structure of the crystal with long-period DQN should approach the band structure of graphene (Fig. 1).
In Fig. 6, all band structures demonstrate Dirac cones. However, in crystals 5-7Bqd1, 5-7Bqd2, and 5-7Bqd3 Dirac cones are shifted relative to the Fermi level by $\sim 0.25 \mathrm{eV}$. The band gaps of all crystals are zero.

It also follows from the results for energy of DQNs (Fig. 7) that with increasing distance between quadrupoles the energy per atom of disclinated graphene crystals decreases. In the limit of infinite distance, it tends to graphene energy.

\section{SUMMARY AND CONCLUSIONS}

Based on the presented results of computer-assisted modeling, the following conclusions can be drawn: (i) Average specific potential energies of graphene crystals containing $\pm \pi / 3$-disclination ensembles in the form of networks are not much higher than graphene energy. In this regard, the appearance and existence of such crystals is quite likely.

(ii) Pseudo-graphenes with tightly packed disclinations, i.e. phagraphene (5-7A) and 5-7B, demonstrate Dirac cone in their band structures (Figs. $3 a$ and 4a). Therefore, they can have properties associated with this feature, such as quantum Hall effects and ultrahigh carrier mobility.

(iii) Pseudo-graphenes with large number of strongly distorted hexagons, crystals 5-7Bg1 and 5-7Bg2, do not demonstrate Dirac cone in their band structures (Figs. $4 b$ and $4 c$ ). 


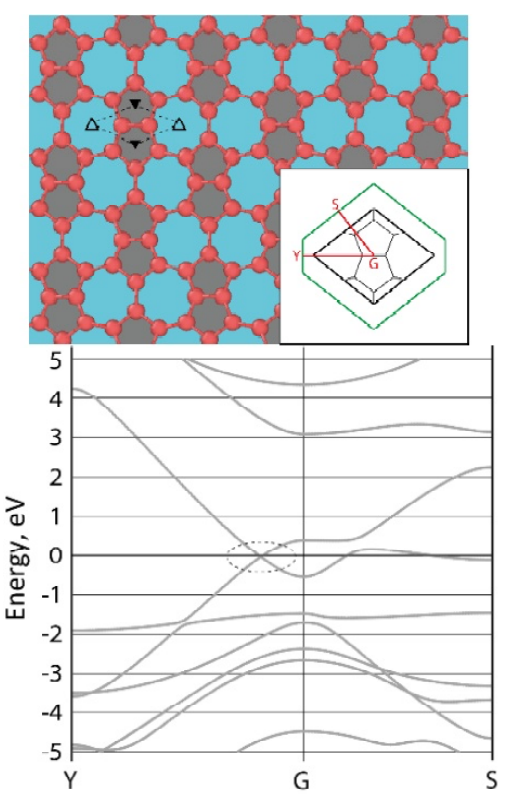

(a) $5-7 \mathrm{~B}$

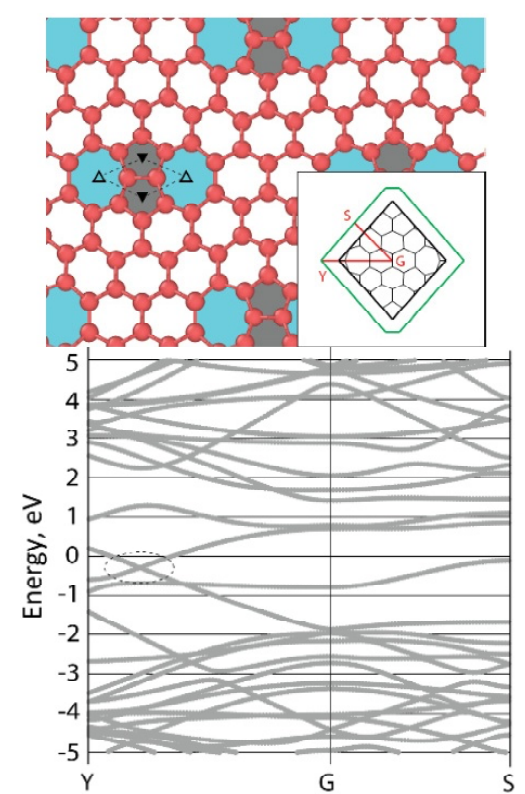

(c) 5 -7Bqd2

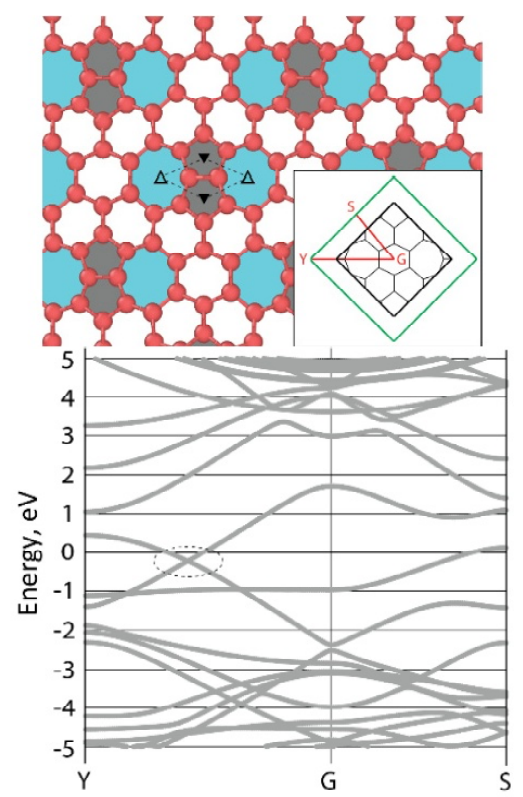

(b) $5-7 \mathrm{~B}$ qd1

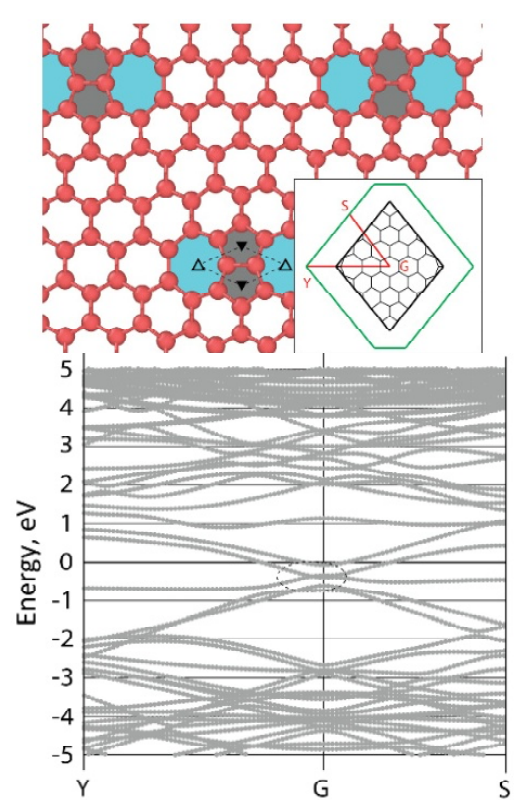

(d) $5-7 \mathrm{Bqd} 3$

Fig. 6. Pseudo-graphene and graphene with networks consisting of disclination quadrupoles and their band structures. In crystal lattices, the disclination quadrupoles are depicted, and the insets show Brillouin zones. Crystals are marked with 5-7B, 5-7Bqd1, 5-7Bqd2, and 5-7Bqd3. Dirac cones are outlined by dashed ellipses.

(iv) Disclinated crystals with weakly distorted hexagons, crystals 5-7Bqd1, 5-7Bqd2, and 5-7Bqd3, demonstarte Dirac cones shifted relative to the Fermi level in their band structures (Figs. 6b-6d).

\section{ACKNOWLEDGEMENTS}

This work was supported by the Ministry of Education and Science of the Russian Federation (Project No 3.3194.2017/4.6).

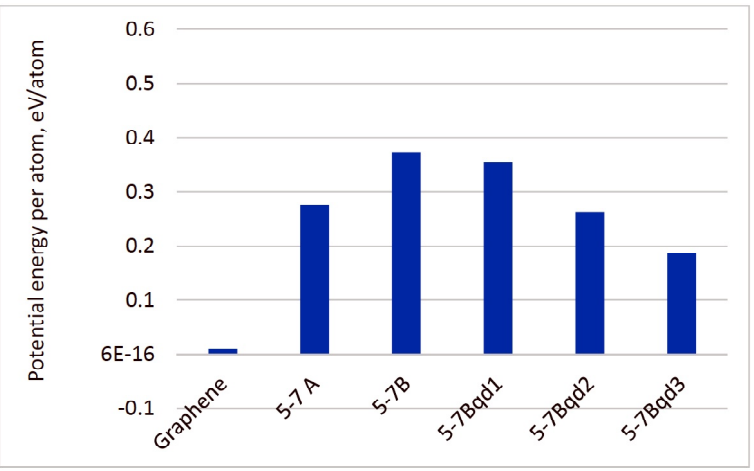

Fig. 7. Average specific potential energies of graphene crystals 5-7B, 5-7Bqd1, 5-7Bqd2, and 5-7Bqd3. 


\section{REFERENCES}

[1] S. Stankovich, D.A. Dikin, G.H. Dommett, K.M. Kohlhaas, E.J. Zimney, E.A. Stach, R.D. Piner, S.T. Nguyen and R.S. Ruoff // Nature 442 (2006) 282.

[2] A.A. Balandin // Nature Materials 10 (2011) 569.

[3] I.W. Frank, D.M. Tanenbaum, A.M. van der Zande and P.L. McEuen // J. Vac. Sci. Technol. B 25 (2007) 2558.

[4] J. Wang, Sh. Deng, Zh. Liu and Zh. Liu // Natl. Sci. Rev. 2(1) (2015) 22.

[5] K.S. Novoselov, A.K. Geim, S.V. Morozov, D. Jiang, M.I. Katsnelson, I.V. Grigorieva, S.V. Dubonos and A.A. Firsov // Nature 438(7065) (2005) 197.

[6] Y.B. Zhang, Y.W. Tan, H.L. Stormer and P. Kim // Nature 438(7065) (2005) 201.

[7] Zh.H. Ni, T.Yu, Y.H. Lu, Y.Y. Wang, Y.P. Feng and Z.X. Shen // ACS Nano 2 (2008) 2301.

[8] F. Hao, D. Fang and Z. Xu // Appl. Phys. Lett. 99 (2011) 041901.

[9] R. Grantab, V.B. Shenoy and R.S. Ruoff // Science 330(6006) (2010) 946.

[10] J. Lahiri, Y. Lin, P. Bozkurt, I.I. Oleynik and M. Batzill // Nature Nanotechnology 5 (2010) 326.

[11] A. Bagri, S.-P. Kim, R.S. Ruoff and V.B. Shenoy // Nano Letters 11 (2011) 3917.

[12] F. Banhart, J. Kotakoski and A.V. Krasheninnikov // ACS Nano 5 (2010) 26.

[13] L. Liu, M. Qing, Y. Wang and S. Chen // J. Mater. Sci. Technol. 31(6) (2015) 599.

[14] J. Kotakoski, A. V Krasheninnikov, U. Kaiser and J. C. Meyer // Phys. Rev. Lett. 106(10) (2011) 105505.

[15] K. Kim, Z. Lee, W. Regan, C. Kisielowski, M. F. Crommie and A. Zettl // ACS Nano 5(3) (2011) 2142.
[16] W.F. Harris // Scientific American 237(6) (1977) 130.

[17] M.A. Rozhkov, A.L. Kolesnikova, I.S. Yasnikov and A.E. Romanov // Low Temperature Physics 44(9) (2018) 918.

[18] A.E. Romanov, M.A. Rozhkov and A.L. Kolesnikova // Letters on Materials 8(4) (2018) 384.

[19] A.E. Romanov and V.I. Vladimirov, Disclinations in Crystalline Solids, In: Dislocations in Solids, ed. by F.R.N. Nabarro. North-Holland, Amsterdam, 1992.

[20] A.E. Romanov, A.L. Kolesnikova, T.S. Orlova, I. Hussainova, V.E. Bougrov and R.Z. Valiev // Carbon 81(1) (2015) 223.

[21] A.L. Kolesnikova, M.A. Rozhkov, I. Hussainova, T.S. Orlova, I.S. Yasnikov, L.V. Zhigilei and A.E. Romanov // Rev. Adv. Mater. Sci. 52(1/2) (2017) 91.

[22] Z. Wang, X. F. Zhou, X. Zhang, Q. Zhu, H. Dong, M. Zhao and A.R. Oganov // Nano Letters 15(9) (2015) 6182.

[23] C.P. Tang and S.J. Xiong // AIP Advances 2(4) (2012) 042147.

[24] http:// lammps.sandia.gov

[25] D.W. Brenner, O.A. Shenderova, J.A. Harrison, S.J. Stuart, B. Ni and S.B. Sinnott // J. Physics: Condensed Matter 14(4) (2002) 783.

[26] E. Polak, Computational Methods in Optimization: A Unified Approach, In: Mathematics in Science and Engineering Ser.: Vol 77 (Academic press, 1971).

[27] http://www.castep.org

[28] J.P. Perdew, K. Burke and M. Ernzerhof // Phys. Rev. Lett. 77(18) (1996) 3865. 